\title{
p-4. Effect of Ventrolateral Nuclei and Subthalamic area to the Spastic State in Cat
}

\author{
Akira Gega, Hideo Sekimoto, Atsuo Okazaki, Kohei Hayashi, \\ Chikayoshi Terada and Yutaka Hori \\ 2nd Department of Surgery, Nara Medical University
}

Tlthough stereotaxic surgery for patients with muscular hypertonic state has performed, adequate target for relieving spastisity has never been found. In some cases of cerebral palsy with rigospasm, spastisity was decreased as well as rigidity by lesion of ventrolateral nuclei, but in some cases spastisity was increased in spite of diminished rigidity. It seems very important to clarify the relationship between spastisity and areas in vicinity of ventrolateral nuclei.

Firstly, subcortical parts of adult cats were stimulated stereotaxically by electrical shocks with $0.1 \mathrm{msec}$. pulse duration, $100 \mathrm{cps}$ and $2-5 \mathrm{~V}$. in bipolar electrode. Motor responses were observed at contralateral gastrocnemius muscle and antetrior tibial muscle. The points that have motor effects on hidelimbs were plotted on the atlas of Jasper and Ajomone-Marsan, and were presented in caudate nuclei, ventrolateral nuclei, ventralis posterior nucleus, centren median nuclei, subthalamic area and internal capsule. The movement by stimulation were different corresponding to the regional points. The movements produced by stimulation of internal capsule were rather distinct and phasic: by stimulation of subthalamic area a little bit phasic: and by stimulation of ventrolateral nuclei rather vague and tonic.

Secondary, the effects on phasic stretch reflex of area vicinity of ventrolateral Iuclei were studied. In spasticity, that seems to be disorder of alpha motor system, clonus occurs most frequently by repetitive phasic muscle stretch. In normal subjects, also, it might be produced easily under ether narcosis. Inserting bipolar clectrode (pole distance, $0.8 \mathrm{~mm}$ ) into the subcortical parts, the effects on the clonus of area in vicinity of ventrolateral nuclei were tested by stimulation with $0.1 \mathrm{msec}$. pulse duration, $100 \mathrm{cps}$ and $5-10 \mathrm{~V}$. During the electrical stimulation in lateral parts of ventrolateral nuclei and subthalamic area clonus suppressed or completely diminished.

\section{p-5. Subthalamic Stimulation Effect on Epileptic Excitability of Neo-, Archi- and Paleocortical Systems}

\author{
Kiyofumi Kobayashi, Yoshikazu Iwata, Jiro Mukawa, \\ Heitaro Mogami and Dennosuke JinNaI \\ Department of Neurosurgery, Osaka University Medical School
}

Electrophysiological study was attempted to find out the existence of facili- 
tatory or inhibitory functions at the subthalamus and near-by structures on the epileptic excitability at the various cortical systems.

Experimental method: Analyses were made on the effect of simultaneous stimulation at the brain stem upon each of three electrically induced afterdischarges from the motor cortex, dorsal hippocampus and amygdala of cats. The criteria for evaluation of facilitation or inhibition were based on the stimulus threshold, discharge duration and pattern.

Results: 1) Subthalamus was facilitatory to the motor cortex, but not to the others. 2) Thalamic structures dorsal to it was not facilitatory to any, but erratically inhibitory to the all. 3) Hypothalamus medial to it had a tendency of facilitation to the all. 4) Cerebral peduncle and substantia nigra lateral to it were facilitatory both to he hippocampus and amygdala, but not to the motor cortex. 5) Midbrain reticular formation caudal to it was facilitatory to the hippocampus, but not to the others. It had rather a tendency of inhibitory function to the motor cortex. 6) Red nucleus was neither facilitatory nor inhibitory.

Conclusions: There were a certain localization of facilitatory and inhibitory functions at the brain stem to the epileptic exhitability at the various cortical systems. The subthalamus, especially the prerubral Forel's H-field was facilitatory to the neocortical system, but not to the archi- and paleocortical systems.

\title{
Q. Diagnostic Methods
}

\section{q-1. Partial and Selective Intracranial Arteriography (IV Report)}

\author{
Hiroyasu Makino, Kenichi Uemura, Kenji Tonozuka, Yasuyuki Harada, \\ Akira Yamamura, Hiroshi Ryu, \& Noriaki NinchoJI \\ 2nd Department of Surgery, Chiba University
}

In the past several years, we have been studying a method of partial and segmental visualization of the intracranial arterial system by internal carotid catheterization. The present communication deals with a method of passing the catheter through the siphon of the internal carotid artery which had been impossible as reported previously. The special graduated needles of our design is used for the introduction of the catheter into the internal carotid artery. After trials with various types of catheter in dogs and human, the flexible Pudenz cardiac catheter was found to be the best for passing through the carotid siphone. This catheter reinforced with a guide wire was first inserted up to the base of the skull where the artery is sharply bent. From here on the catheter is further advanced with the wire left in the same position for the rigid support of the catheter in the cervical portion of the internal carotid artery. By this way in human we were 